\title{
Unraveling the puzzle
}

\author{
Marvin D. Nelson Jr.
}

Received: 15 December 2008 / Accepted: 22 December 2008 / Published online: 23 January 2009

(C) Springer-Verlag 2009

This interesting article reviews the development of the cranial venous system and stresses the point that there is a rich plexus of intradural vessels that can rupture and cause subdural hemorrhage [1].

I have always been puzzled by the classic response that subdural hemorrhage is the result of "tearing of the bridging cortical veins." Shouldn't the bridging vein either rip off the pial surface of the brain or shear off at the point where the vein penetrates the dura? Shouldn't this cause massive subarachnoid hemorrhage? Why should such a vein tear once it has penetrated the dura and not cause the resulting subdural hemorrhage to also flow into the subarachnoid space?

Rupture of smaller intradural vessels makes sense on a lot of levels. These vessels have thinner walls without a muscularis and are often only endothelium and pericytes without much, if any, adventitia. It is also interesting that this plexus of intradural vessels is more prominent in infants than in adults.
Nevertheless, something must happen to the walls of these vessels to allow red blood cells to pass out of the lumen of the vessel into the subdural space. Obviously, trauma remains the most likely and frequent cause. However, the presence of subdural hemorrhage does not tell us whether the action that caused it was accidental, and correlation with the historical account of the injury, the physical examination, and the social services evaluation is necessary before any conclusion can be made about the source of the subdural hemorrhage or the intent of the action that may have caused it.

\section{Reference}

1. Mack J, Squier W, Eastman JT (2009) Anatomy and development of the meninges: implications for subdural collections and CSF circulation. Pediatr Radiol 39. doi: 10.1007/s00247-008-1084-6
Editor's note: see related articles in this issue: doi 10.1007/s00247008-1084-6 and doi 10.1007/s00247-008-1144-y

M. D. Nelson Jr. $(\bowtie)$

Department of Radiology (MS 81), Children's Hospital, 4650 Sunset Blvd.,

Los Angeles, CA 90027, USA

e-mail: mdnelson@chla.usc.edu 